\title{
Simulating nanocrystal-based solar cells: A lead sulfide case study
}

\section{Journal Article}

\section{Author(s):}

Lin, Weyde (D); Yazdani, Nuri (D); Yarema, Olesya (D); Volk, Sebastian (D); Yarema, Maksym (iD; Kirchartz, Thomas; Wood, Vanessa (iD)

Publication date:

2019-12-28

Permanent link:

https://doi.org/10.3929/ethz-b-000389490

Rights / license:

In Copyright - Non-Commercial Use Permitted

Originally published in:

The Journal of Chemical Physics 151(24), https://doi.org/10.1063/1.5129159 


\title{
Title
}

Simulating Nanocrystal-based Solar Cells: A Lead Sulfide Case Study

\section{Authors}

Weyde M. M. Lin ${ }^{1}$, Nuri Yazdani ${ }^{1}$, Olesya Yarema ${ }^{1}$, Sebastian Volk ${ }^{1}$, Maksym Yarema ${ }^{1}$, Thomas Kirchartz ${ }^{2,3}$, Vanessa Wood ${ }^{1}$

\begin{abstract}
Affiliation
${ }^{1}$ Materials and Device Engineering Group, Department of Information Technology and Electrical Engineering, ETH Zurich, 8092 Zurich, Switzerland

${ }^{2}$ IEK-5 Photovoltaik, Forschungszentrum Jülich GmbH, 52425 Jülich, Germany

${ }^{3}$ Faculty of Engineering and CENIDE, University of Duisburg-Essen, Carl-Benz-Str. 199, 47057 Duisburg, Germany
\end{abstract}

\section{Abstract}

Nanocrystal-based solar cells are promising candidates for next generation photovoltaic applications; however, the most recent improvements to the device chemistry and architecture have been mostly trial-and-error based advancements. Due to complex interdependencies among parameters, determining factors that limit overall solar cell efficiency is not trivial. Furthermore, many of the underlying chemical and physical parameters of nanocrystal-based solar cells have only recently been understood and quantified. Here, we show that this new understanding of interfaces, transport, and origin of trap states in nanocrystal-based semiconductors can be integrated into simulation tools, based on 1D drift-diffusion models. Using input parameters measured in independent experiments, we find excellent agreement between experimentally measured and simulated PbS nanocrystal solar cell behavior without having to fit any parameters. We then use this simulation to understand the impact of interfaces, charge carrier mobility, and trap-assisted recombination on nanocrystal performance. We find that careful engineering of the interface between the nanocrystals and the current collector is crucial for an optimal open-circuit voltage. We also show that in the regime of trap-state densities found in $\mathrm{PbS}$ nanocrystal solar cells $\left(\sim 10^{17} \mathrm{~cm}^{-3}\right)$, device performance exhibits strong dependence on 
the trap state density, explaining the sensitivity of power conversion efficiency to small changes in nanocrystal synthesis and nanocrystal thin-film deposition that has been reported in literature. Based on these findings, we propose a systematic approach to nanocrystal solar cell optimization. Our method for incorporating parameters into simulations presented and validated here can be adopted to speed up the understanding and development of all types of nanocrystal-based solar cells. 


\section{Introduction}

Simulation of solar cells plays an important role in understanding and systematic optimization of device design. In industry, silicon and thin film solar cells typically rely on commercial software solutions such as the technical CAD program, Sentaurus Device ${ }^{1}$ or the drift-diffusion simulators SETFOS ${ }^{2}$. Since these commercial software tools are not always financially accessible for academic research groups or may not contain all necessary models (e.g. new absorber materials), here we consider how complex physics (i.e., polaronic transport, charge trapping and detrapping, etc.) can be correctly integrated into a simple one-dimensional, free software. For new absorber materials, such as organics, nanocrystals, and perovskite absorbers, this requires coarse graining the complexity of the material, mapping its physics to simplified scalar parameters which can be directly input into device-level simulations. Given a suitable mapping, drift-diffusion simulations can then be employed to efficiently identify bottlenecks towards enhanced photovoltaic efficiency, and/or parameterize material parameters through measurement on devices (e.g. through Bayesian inference ${ }^{3}$ ).

Here, we focus on how the complex transport in nanocrystal (NC)-based solar cells can be correctly simulated in a 1D drift-diffusion tool. We consider NC thin films because they are promising candidates as the active materials for next generation solar cells due to their bandgap tunability ${ }^{4}$, and solution processability, which can enable light weight and flexible solar cells manufactured at low cost. While there have been many improvements to the NC-based solar cell performance due to changes in device architecture ${ }^{5}$, chemical modifications ${ }^{6}$, and expanding to new material systems ${ }^{7}$, these improvements stem largely from trial-and-error based advancements. To further push the performance of NC-based solar cells towards the theoretical $\operatorname{limits}^{8}$ and develop more complex architectures, it is critical to be able to simulate the device structure and identify sources of performance limitations. 
The output of a simulation however is only as good as the chosen input parameters. Traditionally, it has been non-trivial to simulate NC-based solar cells performance as many parameters are either hard to extract experimentally (e.g. charge-carrier concentration) or have only recently been understood (e.g. the link between doping and trap state formation ${ }^{9}$ ). Previously, NC solar cell simulations were used to study single parameter changes in NC-based solar cells (e.g. changing defect density ${ }^{10}$ or carrier density of heterojunction material ${ }^{11}$ ). In these kinds of simulations, the values of all chosen parameters tend to not be as crucial since the purpose is to document the change resulting from systematic variation of one parameter. However, it is possible that even when changing a single parameter, one misses important effects if other parameters are set such that they dominate.

In this work, we demonstrate how to incorporate the latest understanding of NC-based thin films chemistry ${ }^{12}$ and physics ${ }^{13-15}$ into 1D drift diffusion solar cell simulations using SCAPS (Version 3.3.07). ${ }^{16}$ All simulations parameters are taken from independent measurements and ab-initio calculations on NC materials, and the simulation results show excellent agreement with experimentally measured current-voltage sweeps, temperature-dependent current-voltage (IVT) curves in the dark, and generation-dependent open-circuit voltages. This agreement validates our approach for modeling NC-based solar cells with a simple 1D model, with welldefined effective parameters for NC-metal interfaces and the NC-absorber layer. Furthermore, our simulations provide us with a number of important insights into NC-based solar cells, namely the importance of appropriately engineered interfaces between the nanocrystal and the current collectors and the sensitivity of the device performance to the trap-state density.

\section{Integrating NC Physics into 1D, Drift-Diffusion Models}

Here, we work with the simplest type of NC-based solar cell (the "Schottky-type"), which consists of a metal-semiconductor-metal junction. We use a $100 \mathrm{~nm}$-thick, lead sulfide (PbS) NC thin film cross-linked with ethanedithiol (EDT). Synthesis of the PbS NCs and the 
fabrication of the device has been previous described in detail. ${ }^{17}$ The two metal contacts are indium tin oxide (ITO) and a $1 \mathrm{~nm}$ lithium fluoride (LiF) layer on $100 \mathrm{~nm}$ aluminum (Al).

In Figure 1a, we show the schematic band diagram for the $\mathrm{NC}$ device that is the basis for the simulation. The most important parameters are summarized in Table I. All other parameters used in the simulation are listed in Supplementary Table I.

In trying to implement complex physics into a simple model, we note two aspects in particular that require consideration: (1) the interface between the $\mathrm{NC}$ absorbing layer and the electrodes, and (2) charge carrier densities and dynamics, including charge transport and trapping, along with doping within the NC layer.

First, we consider the interface, specifically the emergence of a charge-transfer dipole. It was recently shown that at a NC-metal interface, NCs can oxidize (or reduce) as a result of the Fermi level equilibration, creating an interfacial dipole located largely across the first NC layer and screened by the dielectric surrounding of the NC thin film ${ }^{12}$. This dipole can be quantified using open-circuit voltage $(\mathrm{OCV})$ measurements before and after deposition of the NC layer on the metal.

Since it was observed that the dipole is localized at the first NC layer(s), here we propose to treat the dipole formed across the interface and the first NC layer as a surface treatment and define an effective work function modification to the metal (Figure 1b). In the case of the present device, we have two modification to the effective work function of the aluminum contact. (1) A thin layer of lithium fluoride $(1 \mathrm{~nm})$ is deposited between the $\mathrm{PbS}$ layer and the aluminum contact. This increases the work function of the electron-accepting contact from 3.99 to $-3.54 \mathrm{eV}^{12}$. (2) As soon as a layer of PbS NCs is deposited, the interfacial dipole ${ }^{12}$ forms and decreases the effective work function $W_{\mathrm{e}}$ by $0.29 \mathrm{eV}$ to $3.79 \mathrm{eV}^{12}$ (See Figure $1 \mathbf{b}$ left). The same happens on the opposite electrode. The effective work function $W_{\mathrm{h}}$ of ITO is shifted due to dipole formation on the surface ${ }^{12}$ from -4.90 to $-4.62 \mathrm{eV}$ (See Figure 1b right). Thus, 
instead of a work function difference of $1.36 \mathrm{eV}(4.9-3.54 \mathrm{eV})$ we have an effective work function difference of $0.91 \mathrm{eV}(4.6-3.79 \mathrm{eV})$. This reduced work function difference already hints at the detrimental effect of the interfacial dipole formation.

Next, we turn to the charge carrier dynamics within the NC thin-film. Most simulation tools are designed with a bulk, crystalline semiconductor in mind with well-defined conduction and valence bands, mobility, doping and free carrier densities. In a NC solid, however, we do not have ballistic transport of charge carriers through conduction and valence bands, but rather temperature activated charge-transfer based transport, with effective charge-carrier mobilities intimately linked to the density of trap states, which stem from oxidized/reduced doped-NCs ${ }^{9}$. We therefore need to consider how to incorporate these parameters in a simulation.

In NCs in the strong quantum confined regime, transport of electrons (and holes) proceeds via phonon-assisted charge transfer between the lowest unoccupied electronic states (highest occupied electronic states) of neighboring $\mathrm{NCs}^{9}$ (See Figure 1c). Thus, the bandgap of the NC thin film is related to the bandgap of the individual NCs. Cyclic voltammetry or energy-resolved electrochemical impedance spectroscopy measurements on PbS-EDT films ${ }^{12}$ can be used to determine the conduction and valence bands ${ }^{12}$. Here, we use a conduction band of $3.79 \mathrm{eV}$ (See Supplementary Figure 5) and a mobility band gap of $1.1 \mathrm{eV}^{18}$.

Due to energetic disorder in the NC thin film, stemming from variation in the individual NC sizes and the presence of trap states, the mobility is not a well-defined quantity; however, we can extract an effective mobility experimentally via time of flight measurements ${ }^{19}$ or, more recently, multiscale simulations ${ }^{9}$. We separately calculate the effective mobilities for electron and holes, $\mu_{e}\left(T, N_{T}, \sigma_{E g}\right)$ and $\mu_{h}\left(T, N_{T}, \sigma_{E g}\right)$, for each set of the discrete temperatures, $T$, density of trap states, $N_{T}$, and values for the standard deviation of the NC band-gap disorder, $\sigma_{E g}$, used in our simulation.

A variety of complementary techniques, including thermal admittance spectroscopy (TAS), 
have determined trap-state densities $N_{\mathrm{T}}{ }^{13}$ on the order of $10^{17} \mathrm{~cm}^{-3}$. The energy depth $E_{\mathrm{T}}$ depends on the NC bandgap (size). The origin of the trap states has long been a question. Recent work has shown that they originate from at least two distinct effects. First, during some thin film preparation approaches, NCs dimerize, resulting in reduced quantum confinement, a smaller band gap energy, and therefore energy states of the dimer that are offset from the energy states of neighboring individual NCs in the film ${ }^{20}$. Thus, the dimer acts as a trap state for charge. Second, oxidation or reduction of an off-stoichiometric/doped NC also leads to trap states, where a trap state is the lowest unoccupied (or highest occupied) energy level of the oxidized (or reduced) doped-NC, shifted in energy from the energy levels of the neighboring nanocrystals (Fig. 1c). Both dimerization and doped NCs result from the colloidal synthesis conditions as well as the conditions during film deposition ${ }^{21,22}$. Here we select a trap density $N_{\mathrm{T}}=10^{17} \mathrm{~cm}^{-3}$, and an energy depth $E_{\mathrm{T}}=250 \mathrm{meV}$ from the conduction band, following the calculations by Yazdani et al. ${ }^{9}$

Next, we consider doping of the NC thin film. To date, doping has been primarily achieved through doping of the individual NCs. These doped carriers, however, experience a large energetic barrier to leave the doped-NC and become a 'free carrier' within the thin-film. ${ }^{9}$ This energetic barrier is equivalent to the trap-depth, $E_{\mathrm{T}}$, discussed above. We therefore have no shallow dopants in the system and set the number of shallow donors/acceptors $N_{\mathrm{D} \text {,shallow }}$ and $N_{\mathrm{A} \text {,shallow }}$ to $0 \mathrm{~cm}^{-3}$. We include doping in the thin-film through the inclusion of acceptor-like with an energetic depth given by $E_{\mathrm{T}}$. Given the one-to-one correspondence between dopedNCs and trap-states, set $N_{\mathrm{A}, \text { deep }}=N_{\mathrm{T}}=10^{17} \mathrm{~cm}^{-3}$ and $N_{\mathrm{D} \text {,deep }}=0 \mathrm{~cm}^{-3}$.

Recombination in NC occurs through trap-mediated Shockley-Read-Hall (SRH) recombination. SRH recombination is a multi-phonon recombination process, where rates in the low and high temperature limits can be calculated ${ }^{23}$. The overlap of the wavefunction between vibrational excited states of a lower lying electronic state (e.g. a defect) with a higher lying electronic 
state (that is not or not much vibrationally excited) gives us the capture coefficient $\varphi,{ }^{18}$ which we have previously determined by transient photovoltage measurements to be $\varphi=10^{9} \mathrm{~cm}^{-3} \mathrm{~s}^{-1} .{ }^{17}$ In SCAPS, the capture coefficient cannot directly be added as input; instead, a thermal velocity $v_{\text {th }}$ and capture cross section $\sigma$ are needed from which the capture coefficient is determined: $\varphi=v_{\text {th }} \sigma$. We therefore set the capture cross section to $\sigma=10^{-13} \mathrm{~cm}^{2}=10 \mathrm{~nm}^{2}$ (i.e., which is the size of a $\mathrm{NC}$ and its nearest neighbors and is thus a value consistent with the picture that charge traps on individual NCs or dimers) and can approximate the thermal velocity as $v_{\text {th }}=$ $10^{4} \mathrm{~cm} / \mathrm{s}$.

Surface recombination is also known to play an important part $^{24}$ in the overall performance of a solar cell. While this process has been extensively studied in other solar cell technologies (such as silicon ${ }^{25}$, perovskite ${ }^{26}$ or organic solar cells ${ }^{27}$ ), not much is known about the surface recombination in $\mathrm{PbS}$ NCs solar cells. The surface recombination is described through SRH statistics as the recombination is mediated by surface or interface trap states ${ }^{28}$. Fundamentally, the recombination will therefore be dependent on the electron-phonon coupling between the charge carriers and the surface traps. For the initial simulations, we set a surface recombination rate for electrons of $10^{3} \mathrm{~cm} / \mathrm{s}$. This is somewhat smaller than for silicon, where surface recombination is $10^{5}-10^{6} \mathrm{~cm} / \mathrm{sec}^{25}$ or for organics $\left(10^{4} \mathrm{~cm} / \mathrm{s}\right)^{27}$, but is a value where the recombination within the $\mathrm{NC}$ absorber layer dominates over the surface recombination.

\section{Experimental Validation of Simulation Approach}

The most common measurement to characterize a solar cell is a current-voltage sweep under AM1.5 illumination (light-IV). Comparing the measured device (Figure 2a) light-IV curve to the simulated device (Figure $\mathbf{2 b}$ ) shows that the two are in good agreement.

However, relying on a good match between the light-IV curves to assess the quality of a simulation and the input parameters can be misleading. A large number of parameters are used in the simulation, and multiple combinations of parameters can lead to a very similar looking 
light-IV curve. Other measurements beside the light-IV sweep should thus be used to validate the simulation results.

In Bozyigit et al. ${ }^{18}$, temperature-dependent IV (IVT) measurements in the dark were used to study carrier transport, trapping, and recombination. We therefore compare a measured IVT (Figure 2c) with a simulated IVT (Figure 2d) and find good agreement. Specifically, we note agreement in several features. First, the comparable increase in current as a function of temperature for all voltages shows that the temperature-dependence of the mobility has been successfully incorporated into the simulation. Second, the kink in the IVT originates from a change in dominating loss mechanism is visible in both the experimental and simulated IVT. At lower voltage $(<1 \mathrm{~V})$, the current is limited by trap-assisted recombination; at higher voltage $(>1 \mathrm{~V})$, the current is limited by external series resistance.

To study recombination dynamics in NC-solids, we previously used transient photovoltage measurements ${ }^{17}$. How the open-circuit voltage $\left(V_{\text {oc }}\right)$ varies as a function of carrier generation rate $(G)$ depends on the density of recombination centers (i.e., trap states), as at $V_{\text {oc }}$ the recombination rate is equal to the generation rate. Comparing the $V_{\mathrm{oc}}$ vs. $G$ extracted from experiment (Figure 2e) and that simulated here (Figure 2f), we see that in both the open-circuit voltage: (1) increases with increasing generation rate and (2) decreases with increasing temperature. The experimental open-circuit voltage saturates at $0.57 \mathrm{~V}$, while the simulated curve saturates as $\sim 0.83 \mathrm{~V}$ (corresponding to difference between $W_{\mathrm{e}}$ and $W_{\mathrm{h}}$, see Supplementary Materials). This saturation occurs because $V_{\mathrm{OC}}$ approaches the built-in voltage $\left(V_{\mathrm{bi}}\right)$ of the device. Artificially decreasing the $V_{\text {bi }}$ in the simulation to yield a saturation at $0.57 \mathrm{~V}$ leads to a $V_{\mathrm{OC}}$ that is too low at room temperature. This highlights that our simulation with a finite number of parameters cannot capture every performance aspect under all possible conditions, it describes the behavior of our NC-based metal-semiconductor-metal well, which is impressive given that no fitting is performed and that all input parameters come from independent experimental 
measurements. Of course, measurements that capture parameters as a function of light intensity, wavelength (such as external quantum efficiency measurement), film thickness, or a combination of these could be used to enable further verification.

\section{Discussion}

Having experimentally validated the simulation and the parameter set, we now use it to gain insight into three aspects of NC-based devices. Keeping all other parameters the same, we repeat the simulations, systematically changing (1) the magnitude of the interface dipole, (2) the trap state density, and (3) the mobility of the NC thin film.

$V_{\text {oc }}$ in a metal-semiconductor-metal junction will be limited by the energy offset between the two contacts. Thus, careful selection of the work functions of the two electrodes is important for device performance. In the case of a dipole $\left(\Delta_{\text {dipole }}\right)$ that decreases the offset between the two metal contacts, increasing the magnitude of the $\Delta_{\text {dipole }}$ will only change the short circuit current by a small amount (Figure 3a), but the open-circuit voltage will decrease almost linearly (Figure 3b). This leads to decreasing fill factor (Figure 3c) and power conversion efficiencies (Figure 3d). When the $\Delta_{\text {dipole }}$ reaches $\sim-0.8 \mathrm{eV}$ (i.e. $W_{\mathrm{e}} \approx W_{\mathrm{h}}-\Delta_{\text {dipole }}$ ), the device is no longer a rectifying diode and the solar cell performance parameters become meaningless.

Therefore, a careful selection of the electrode work function and/or adding self-assembled monolayers of organic molecules to shift the work function ${ }^{12}$ is crucial when designing a NCbased solar cell. Another option is to change the surface chemistry of the PbS NCs. For example, Volk et al. ${ }^{12}$ showed that changing the ligand from EDT to $\mathrm{SCN}^{-}$lowers the $\Delta_{\text {dipole }}$ resulting in effective work function close to the ITO work function. Leveraging a heterojunction solar cell design can further improve the $V_{\mathrm{OC}}{ }^{29}$ By matching the work function of the metals to the conduction and valence bands of the NCs, it is possible to design ideal metal-semiconductor as has been demonstrated for diodes made with the $2 \mathrm{D}$ semiconductors ${ }^{30}$. 
We next investigate the impact of trap state density $\left(N_{\mathrm{T}}\right)$ on device performance. Intuitively, a smaller $N_{\mathrm{T}}$ should lead to less recombination and therefore better performance. However, this turns out to be true only for a narrow range of trap state densities. For a mobility of $0.18 \mathrm{~cm}^{2} / \mathrm{Vs}$ (orange dashed line), for $N_{\mathrm{T}} \lesssim 10^{17} \mathrm{~cm}^{-3}$, decreasing the trap state density does not further improve the performance (Figure 3e-h). In the range between $N_{\mathrm{T}}=10^{17}-10^{18} \mathrm{~cm}^{-3}$ (which corresponds to 1 to $10 \%$ of NCs in the solid acting as traps), $J_{\text {sc }}$ shows a strong dependence on $N_{\mathrm{T}}$, such that a small change in trap state density can have a large influence on the device performance. Interestingly, measurements of trap state densities show that PbS NC solar cells have trap state densities of this order of magnitude ${ }^{10,13,31-33}$. Our simulations thus explain the practical observation that the efficiency of PbS solar cells can vary dramatically with small changes in external condition (age/purity of precursor, storage time, humidity during film deposition ${ }^{22}$ ) that can influence the number of trap states in the $\mathrm{NC}^{9,20}$.

Varying mobility shows that a higher density of trap states are tolerated in devices with higher mobility. For low $N_{\mathrm{T}}$, the short circuit current is almost independent of the mobility (Figure 3e). The $V_{\mathrm{OC}}$ on the other hand shows an improvement with smaller mobility in the same range (Figure 3f) due to lower recombination. We note that the extent of the impact of the mobility is strongly dependent on whether recombination in the NC layer or surface recombination dominates (See Supplementary Figure 3). ${ }^{34}$

In short, in NC-solar cells, it is important to optimize both the NC-contact interface to optimize the surface dipole configurations and minimize surface recombination. After which, it is important to increase the mobility and decrease the number of trap states (or carrier coupling to these trap states ${ }^{14}$ ), ideally developing thin films with independent control over mobility and free carrier densities (at present linked to trap states).

In conclusion, we have shown that it is possible to capture much of the complex physics occurring in a NC-based solar cell with a simple 1D drift-diffusion simulation that is correctly 
parametrized. This can be done without fitting of any simulation parameters, but rather systematic measurements or calculation of the surface dipoles, charge mobility, trap state densities. In addition, our simulation enabled us to gain important insight into significant performance limiting parameters and outline possible solutions to overcome these limitations. These insights can help to understand the more complex heterojunction PbS NC-based solar cells that show generally better performance than the metal-semiconductor-metal described in this work.

\section{Supplementary Materials}

A complete list of all the parameters used as well as further explanation to some of the parameters can be found in the supplementary materials.

\section{Acknowledgement}

The authors thank Prof. Dr. Marc Burgelman for his guidance and explanation in using SCAPS. The authors acknowledge support from the Swiss National Science Foundation through the Quantum Sciences and Technology NCCR and an ETH Research Grant \#42-12-2. 


\section{References}

${ }^{1}$ Synopsys, https://www.synopsys.com/silicon/tcad/device-simulation/sentaurus-device.html (2019).

${ }^{2}$ FLUXiM AG, https://www.fluxim.com/setfos-intro (2019).

${ }^{3}$ R.E. Brandt, R.C. Kurchin, V. Steinmann, D. Kitchaev, C. Roat, S. Levcenco, G. Ceder, T. Unold, and T. Buonassisi, Joule 1, 843 (2017).

${ }^{4}$ M.A. Hines and G.D. Scholes, Adv. Mater. 15, 1844 (2003).

${ }^{5}$ C.-H.M. Chuang, P.R. Brown, V. Bulović, and M.G. Bawendi, Nat. Mater. 13, 796 (2014).

${ }^{6}$ M. Liu, O. Voznyy, R. Sabatini, F.P. García de Arquer, R. Munir, A.H. Balawi, X. Lan, F. Fan, G. Walters, A.R. Kirmani, S. Hoogland, F. Laquai, A. Amassian, and E.H. Sargent, Nat. Mater. 16, 258 (2017).

${ }^{7}$ E.M. Sanehira, A.R. Marshall, J.A. Christians, S.P. Harvey, P.N. Ciesielski, L.M. Wheeler, P. Schulz, L.Y. Lin, M.C. Beard, and J.M. Luther, Sci. Adv. 3, eaao4204 (2017).

${ }^{8}$ National Center for Photovoltaics (NCPV) at NREL, (2019).

${ }^{9}$ N. Yazdani, S. Andermatt, M. Yarema, V. Farto, M.H. Bani-Hashemian, S. Volk, W. Lin, O. Yarema, M. Luisier, and V. Wood, arXiv:1909.09739 (2019).

${ }^{10}$ Y.-B. Zhao, M. Liu, O. Voznyy, B. Sun, P.-C. Li, H. Kung, O. Ouellette, M.-J. Choi, Z.-H. Lu, F.P. García de Arquer, and E.H. Sargent, Nano Energy 63, 103876 (2019).

${ }^{11}$ M. Liu, F.P.G. de Arquer, Y. Li, X. Lan, G.-H. Kim, O. Voznyy, L.K. Jagadamma, A.S. Abbas, S. Hoogland, Z. Lu, J.Y. Kim, A. Amassian, and E.H. Sargent, Adv. Mater. 28, 4142 (2016).

${ }^{12}$ S. Volk, N. Yazdani, O. Yarema, M. Yarema, D. Bozyigit, and V. Wood, J. Phys. Chem. Lett. 9, 7165 (2018). 
${ }^{13}$ D. Bozyigit, S. Volk, O. Yarema, and V. Wood, Nano Lett. 13, 5284 (2013).

${ }^{14}$ N. Yazdani, D. Bozyigit, K. Vuttivorakulchai, M. Luisier, I. Infante, and V. Wood, Nano Lett. 18, 2233 (2018).

${ }^{15}$ D. Bozyigit, N. Yazdani, M. Yarema, O. Yarema, W.M.M. Lin, S. Volk, K.

Vuttivorakulchai, M. Luisier, F. Juranyi, and V. Wood, Nature 531, 618 (2016).

${ }^{16}$ M. Burgelman, P. Nollet, and S. Degrave, Thin Solid Films 361-362, 527 (2000).

${ }^{17}$ W.M.M. Lin, D. Bozyigit, O. Yarema, and V. Wood, J. Phys. Chem. C 120, 12900 (2016).

${ }^{18}$ D. Bozyigit, W.M.M. Lin, N. Yazdani, O. Yarema, and V. Wood, Nat. Commun. 6, 6180 (2015).

${ }^{19}$ N. Yazdani, D. Bozyigit, O. Yarema, M. Yarema, and V. Wood, J. Phys. Chem. Lett. 5, 3522 (2014).

${ }^{20}$ R.H. Gilmore, Y. Liu, W. Shcherbakov-Wu, N.S. Dahod, E.M.Y. Lee, M.C. Weidman, H. Li, J. Jean, V. Bulović, A.P. Willard, J.C. Grossman, and W.A. Tisdale, Matter 1, 250 (2019).

${ }^{21}$ A.R. Kirmani, G.H. Carey, M. Abdelsamie, B. Yan, D. Cha, L.R. Rollny, X. Cui, E.H. Sargent, and A. Amassian, Adv. Mater. 26, 4717 (2014).

${ }^{22}$ A.R. Kirmani, A.D. Sheikh, M.R. Niazi, M.A. Haque, M. Liu, F.P.G. de Arquer, J. Xu, B. Sun, O. Voznyy, N. Gasparini, D. Baran, T. Wu, E.H. Sargent, and A. Amassian, Adv. Mater. 30, 1801661 (2018).

${ }^{23}$ A. Schenk, Solid. State. Electron. 35, 1585 (1992).

${ }^{24}$ O.J. Sandberg, A. Sundqvist, M. Nyman, and R. Österbacka, Phys. Rev. Appl. 5, 044005 (2016).

${ }^{25}$ A. Cuevas, P.A. Basore, G. Giroult-Matlakowski, and C. Dubois, J. Appl. Phys. 80, 3370 
(1996).

${ }^{26}$ I. Zarazua, G. Han, P.P. Boix, S. Mhaisalkar, F. Fabregat-Santiago, I. Mora-Seró, J.

Bisquert, and G. Garcia-Belmonte, J. Phys. Chem. Lett. 7, 5105 (2016).

${ }^{27}$ O.J. Sandberg, M. Nyman, and R. Österbacka, Phys. Rev. Appl. 1, 024003 (2014).

${ }^{28}$ S.M. Sze and K.K. Ng, Physics of Semiconductor Devices (John Wiley \& Sons, Inc., Hoboken, NJ, USA, 2006).

${ }^{29}$ P.R. Brown, R.R. Lunt, N. Zhao, T.P. Osedach, D.D. Wanger, L.-Y. Chang, M.G.

Bawendi, and V. Bulović, Nano Lett. 11, 2955 (2011).

${ }^{30}$ Y. Liu, J. Guo, E. Zhu, L. Liao, S.-J. Lee, M. Ding, I. Shakir, V. Gambin, Y. Huang, and X. Duan, Nature 557, 696 (2018).

${ }^{31}$ A.H. Ip, S.M. Thon, S. Hoogland, O. Voznyy, D. Zhitomirsky, R. Debnath, L. Levina, L.R. Rollny, G.H. Carey, A. Fischer, K.W. Kemp, I.J. Kramer, Z. Ning, A.J. Labelle, K.W. Chou, A. Amassian, and E.H. Sargent, Nat. Nanotechnol. 7, 577 (2012).

${ }^{32}$ Z. Jin, A. Wang, Q. Zhou, Y. Wang, and J. Wang, Sci. Rep. 6, 37106 (2016).

${ }^{33}$ J. Tang, K.W. Kemp, S. Hoogland, K.S. Jeong, H. Liu, L. Levina, M. Furukawa, X. Wang, R. Debnath, D. Cha, K.W. Chou, A. Fischer, A. Amassian, J.B. Asbury, and E.H. Sargent, Nat. Mater. 10, 765 (2011).

${ }^{34}$ T. Kirchartz, B.E. Pieters, K. Taretto, and U. Rau, Phys. Rev. B 80, 035334 (2009). 
Figures and Tables
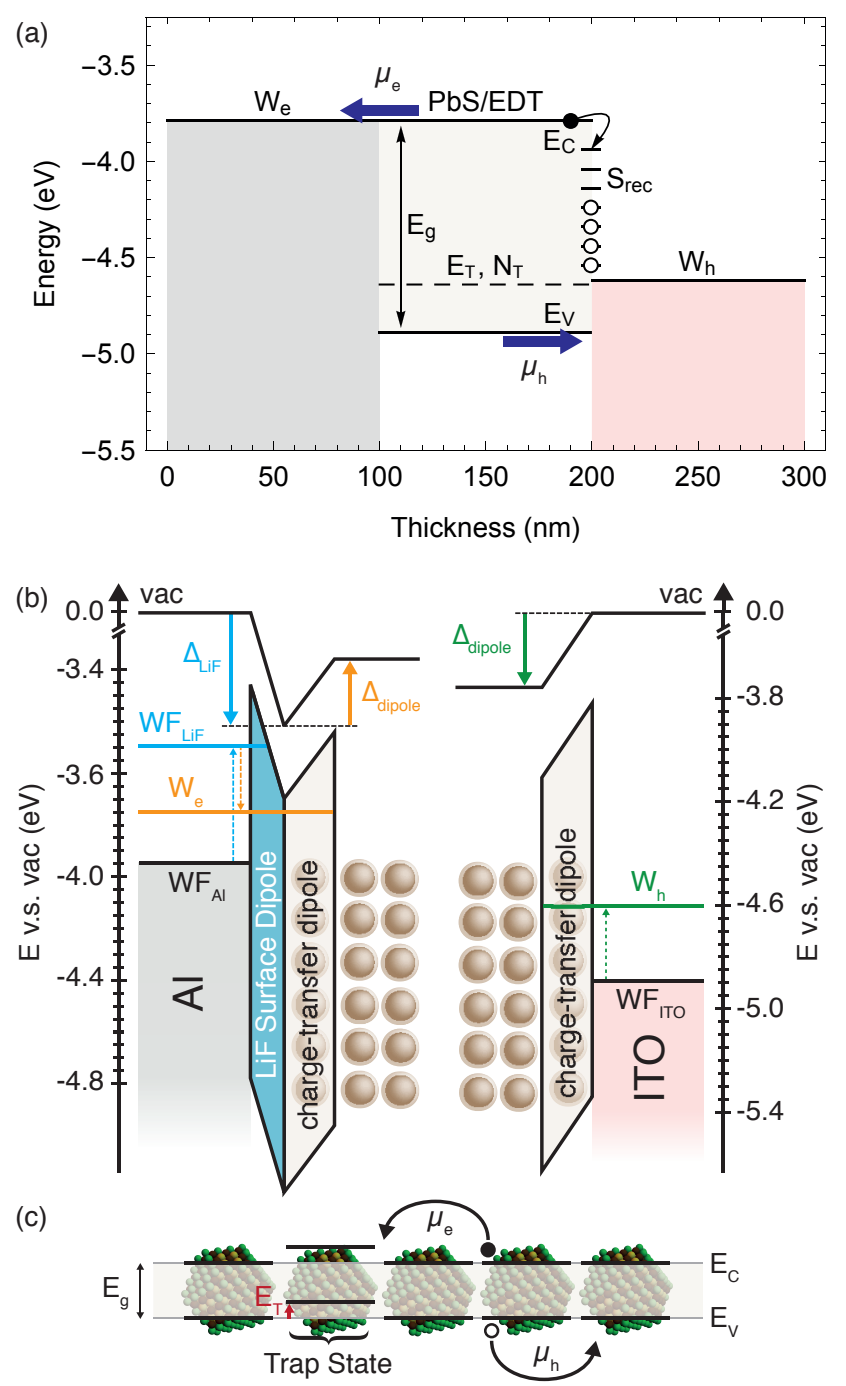

Figure 1 a) Schematic band diagram of a nanocrystal solar cell as input into the simulation tool. Important parameters include the work functions of the electron- and hole-accepting contacts $\left(W_{\mathrm{e}}\right.$ and $\left.W_{\mathrm{h}}\right)$, the position of the conduction and valence band edges $\left(E_{\mathrm{c}}\right.$ and $\left.E_{\mathrm{v}}\right)$ in the nanocrystal (NC) thin film, the effective electron and hole mobilities in the nanocrystal fields $\left(\mu_{\mathrm{e}}\right.$ and $\mu_{\mathrm{h}}$ ), and the surface recombination at the contacts, $S_{\text {rec. }}$ Values for these parameters are listed in Table I. b) Dipoles form at the aluminum (Al) - lithium fluoride (LiF) - lead sulfide $(\mathrm{PbS}) \mathrm{NC}$ interfaces (left) and at the PbS NC - ITO interface (right), leading to shifts in the work function. These dipoles are included in the simulation by defining effective work functions for the two contacts, $W_{\mathrm{e}}$ and $W_{\mathrm{h}}$. c) Schematic depiction of electron and hole transport through a $\mathrm{PbS}$ nanocrystal layer. Charge transport occurs through phonon-assisted charge transfer, a thermally activated hopping process for which effective mobilities $\left(\mu_{\mathrm{e}}\right.$ and $\left.\mu_{\mathrm{h}}\right)$ can be calculated. The values for $E_{\mathrm{c}}$ and $E_{\mathrm{v}}$ used in the simulation tool come from the lowest unoccupied and highest occupied quantized states of the individual NCs. NCs with energy levels that are off-set from their neighbors act as trap states. The energy difference between the quantized states of the individual NCs is the energy depth of the trap, $E_{\mathrm{T}}$. These traps occur with a number density $N_{\mathrm{T}}$. 
Table I. Overview of important parameters and values used in the simulation. The electron and hole mobilities are temperature dependent (see Yazdani et al. ${ }^{9}$ for more information). The values here are given at $300 \mathrm{~K}$. All other parameters used in the simulations can be found in Supplementary Table I of the Supplementary Materials.

\section{Name Variable Value Unit Source}

PbS -EDT

\begin{tabular}{|c|c|c|c|c|}
\hline bandgap & $E_{\mathrm{g}}$ & 1.1 & $\mathrm{eV}$ & Bozyigit e \\
\hline electron affinity & $E_{\mathrm{C}}$ & 3.79 & $\mathrm{eV}$ & $\begin{array}{l}\text { See supple } \\
\text { tary mater }\end{array}$ \\
\hline shallow doping density & $N_{\mathrm{D}} / N_{\mathrm{A}}$ & 0 & $\mathrm{~cm}^{-3}$ & See text. \\
\hline mobilities e/h at $300 \mathrm{~K}$ & $\mu_{\mathrm{e}} / \mu_{\mathrm{h}}$ & 0.18 & $\mathrm{~cm}^{2} / \mathrm{Vs}$ & See text. \\
\hline $\begin{array}{l}\text { energy level with respect } \\
\text { to } E_{\mathrm{V}}\end{array}$ & $E_{\mathrm{T}}$ & 0.25 & $\mathrm{eV}$ & See text. \\
\hline $\begin{array}{l}\text { trap state density/deep p- } \\
\text { doping density }\left(N_{\mathrm{A}, \text { deep }}\right)\end{array}$ & $N_{\mathrm{T}}$ & $\sim 10^{17}$ & $\mathrm{~cm}^{-3}$ & See text. \\
\hline $\begin{array}{l}\text { deep n-doping density } \\
\left(N_{D, \text { deep }}\right)\end{array}$ & - & 0 & $\mathrm{~cm}^{-3}$ & See text. \\
\hline thermal velocity & $v_{\text {th }}$ & $10^{4}$ & $\mathrm{~cm} \mathrm{~s}^{-1}$ & See text. \\
\hline capture cross section $\mathrm{e} / \mathrm{h}$ & $\sigma_{\mathrm{e}} / \sigma_{\mathrm{h}}$ & $10^{-13}$ & $\mathrm{~cm}^{2}$ & See text. \\
\hline
\end{tabular}

Al/LiF - Dipole

$\begin{array}{lllll}\text { metal work function } & W_{\mathrm{e}} & 3.79 & \mathrm{eV} & \text { Volk et al. }^{12}\end{array}$

ITO - Dipole

$\begin{array}{lllll}\text { metal work function } & W_{\mathrm{h}} & 4.62 & \mathrm{eV} & \text { Volk et al. }^{12} \\ \text { surface recombination ve- } & S_{\text {rec }} & \sim 10^{3} & \mathrm{~cm} / \mathrm{s} & \text { See text. }\end{array}$

locity electrons 

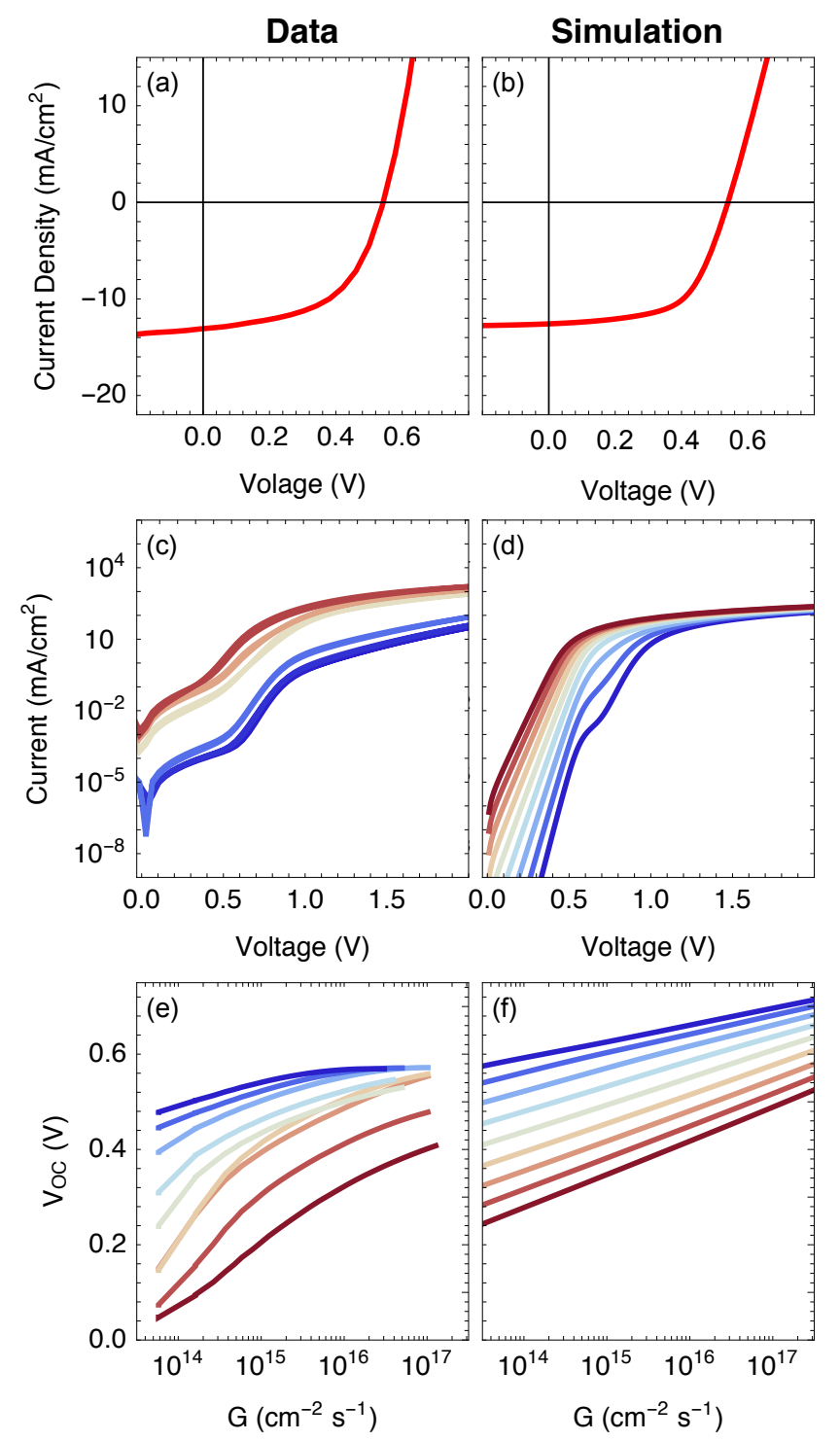

Figure 2. $(a, b)$ Measured and simulated current-voltage sweep under AM1.5 illumination at $300 \mathrm{~K}$. (c,d) Measured and simulated current-voltage sweeps in the dark for temperatures from 180 and 340 K. (e,f) Measured and simulated open-circuit voltage as a function of the generation rate. 


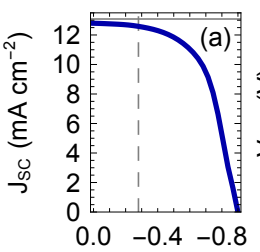

$\Delta_{\text {dipole }}(\mathrm{eV})$
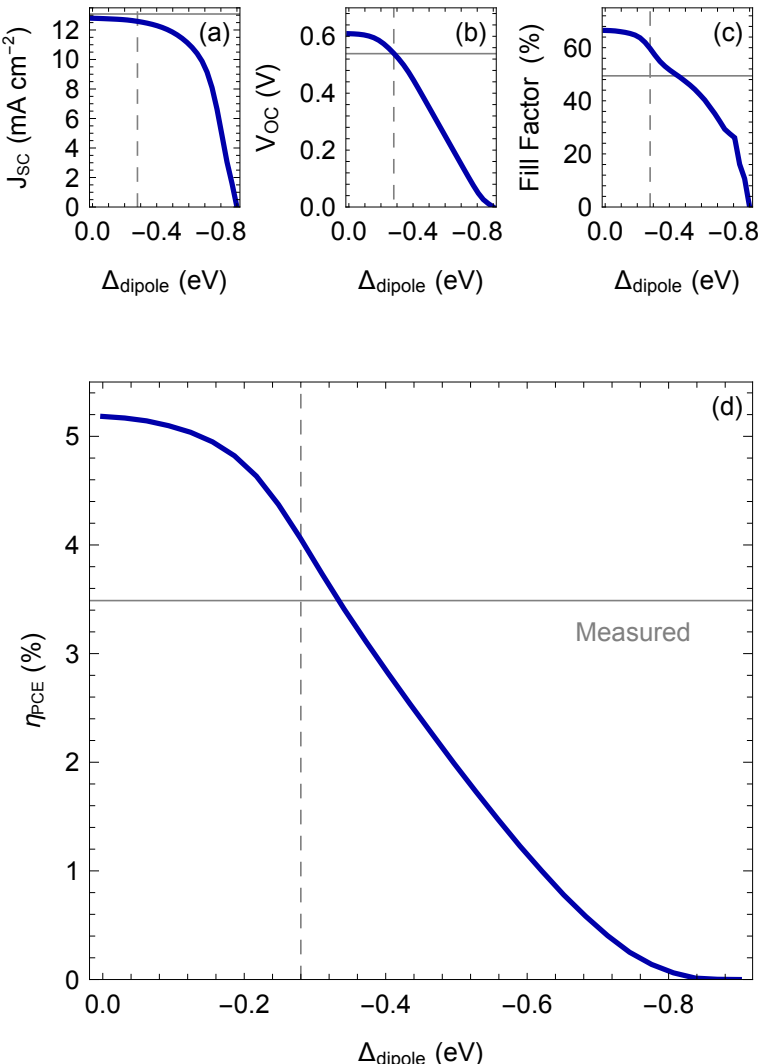
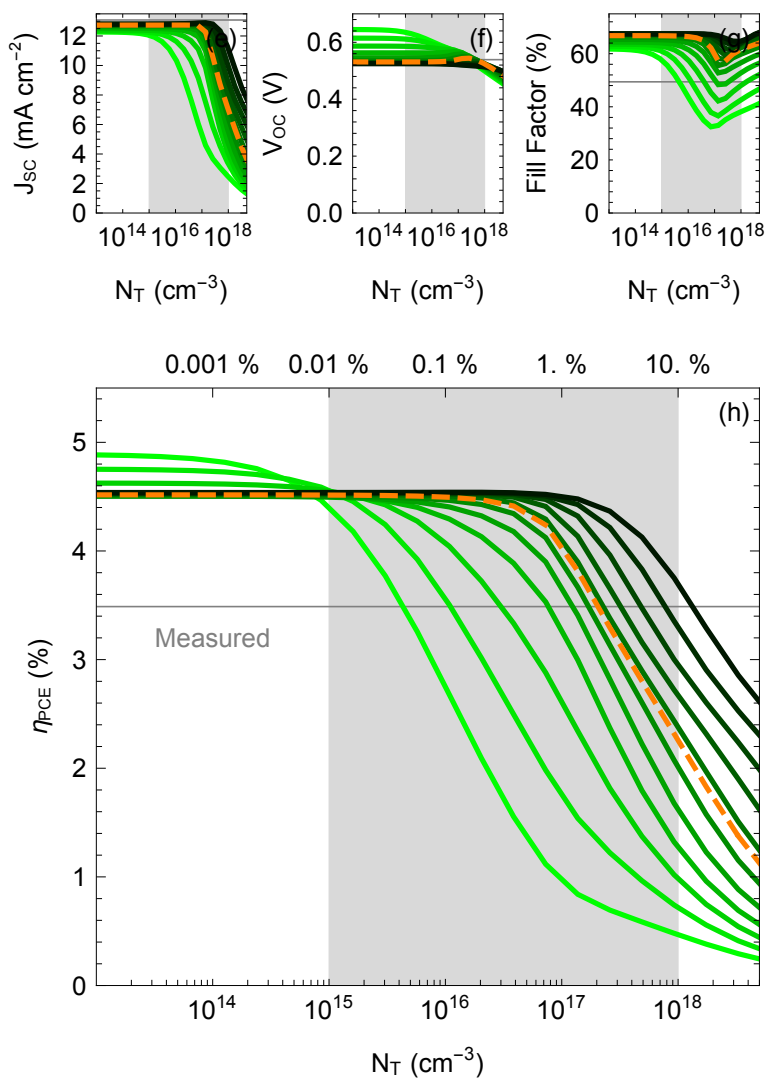

Figure 3. Simulated a) short-circuit current, b) open-circuit voltage, c) fill factor and d) efficiency as a function of dipole height at the PbS NC-ITO interface. The measured dipole $(0.28 \mathrm{eV})$ is marked with a grey, dashed line. The solid line indicates the values extracted from the experimental data (Figure 2a). Simulated e) short-circuit current, f) open-circuit voltage, g) fill factor and h) efficiency as function of the trap state density $N_{\mathrm{T}}$ for mobilities from $10^{-3}$ to $10 \mathrm{~cm}^{2} / \mathrm{Vs}$ (green to black). The dashed orange curve indicates a mobility of $0.18 \mathrm{~cm}^{2} / \mathrm{Vs}$. The grey shared area marks the literature values for reported trap state densities ${ }^{10,13,31-33}$. 